\title{
Numerical study on temperature field of the sandwich plate defects based on MATLAB
}

\author{
Ruoyang Zhou ${ }^{1}$, Xiaoxiong Zha ${ }^{1, *}$, and Chao Wang ${ }^{1}$ \\ ${ }^{1}$ Shenzhen Graduate School, Harbin Institute of Technology, Shenzhen 518055, Guangdong, China
}

\begin{abstract}
As for the defects of the plate whose length and width are more than 8 times larger than the thickness direction, the finite difference numerical calculations are carried out by using the software of MATLAB. The surface temperature of defects in different positions, different thickness defects, different heating heat flow and heating time, and defects under defects are analyzed correspondingly by the program. According to the temperature difference results of the flawless surface and the flawed surface, the feasibility of infrared thermal imaging to detect the defects of the sandwich plate is analyzed to ensure the processing quality of the sandwich plate.
\end{abstract}

\section{Introduction}

The type of detection involves non-destructive testing and destructive testing ${ }^{[1]}$. In actual construction sites, for the purpose of keeping members' original state, nondestructive testing was often used. Non-destructive testing has been widely used in different detection based on the principles of acoustic, electricity, magnetism, optics and heat. According to the principle of acoustic, we have developed ultrasonic testing technology, acoustic emission testing technology, laser ultrasonic testing technology and so on; due to the principle of electricity, we have developed many sensing technology; according to the principle of magnetism, we have developed magnetic particle testing technology, eddy current testing technology, magnetic memory testing technology and so on; given that the principle of optics and heat, we have developed visual detection, penetration detection, radar detection, infrared thermal imaging detection, ray detection and so on. Infrared thermal imaging detection is based on the infrared radiation energy of the object. There is little difference for the surface emissivity of the building, which can be approximately regarded as the detection of the surface temperature difference. The thickness of the sandwich plate is thin, and the defects of the plate have directly impact on the stress performance. It is necessary to verify the quality of the plate to ensure the safety of the plate. Aiming at defects of the plate whose length and width are more than 8 times larger than the thickness direction $^{[2]}$, the finite difference numerical calculations are carried out by using the software of MATLAB in this study. According to the results of the calculation, we can obtain the response of the surface temperature difference to verify the feasibility of infrared thermal imaging detection.

\section{Principle}

According to Stefan-Boltzmann law ${ }^{[3]}$, the relationship between the total radiant power of various wavelengths emitted by the black body in unit area and the thermodynamic temperature $\mathrm{T}$ is quadratic

$$
W=\sigma T^{4} \text {. }
$$

However, the ideal black body is almost non-existent, and most of them will exist in the form of an approximate gray body, which is also the principle of the operation of the infrared thermography. Since the emissivity of different buildings without big difference, the infrared thermal imaging detection of buildings can be approximately regarded as surface temperature difference detection $^{[4]}$

$$
W=\rho \sigma T^{4} \text {. }
$$

When studying the time-dependent unsteady heat conduction, the analytical solution can be obtained by studying the single-layer wall. However, the exact analytical solution for the multi-layer wall is complex. Since it is difficult to obtain an analytical solution when it involves transcendental equation. The finite difference method can be used to solve the numerical solution. The type of finite difference method for cargo without inner thermal source and no convection term involves three difference schemes, explicit scheme, implicit scheme and Crank-Nicolson scheme ${ }^{[5]}$, which are shown in Fig. 1.

\footnotetext{
* Corresponding author: zhahero@126.com
} 


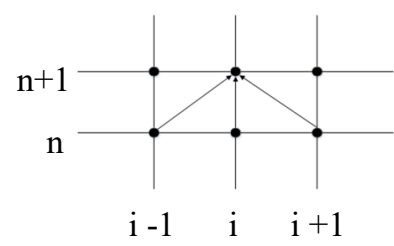

(a) explicit scheme

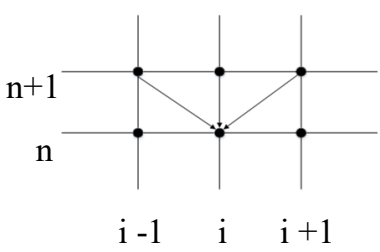

(b) implicit scheme

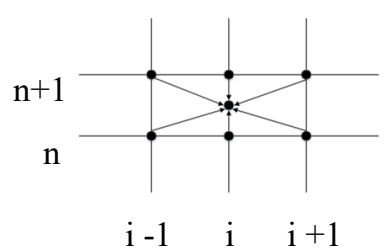

(c) Crank-Nicolson scheme

Fig. 1. Difference scheme of finite difference method

As shown in Fig. 2., it is assumed that there are defects of the plate whose length and width are more than 8 times larger than the thickness direction, and we can almost regard the defective layer as single-layer of wall. Therefore, this kind of heat conduction is equal to the unsteady heat conduction of N-layer plate.

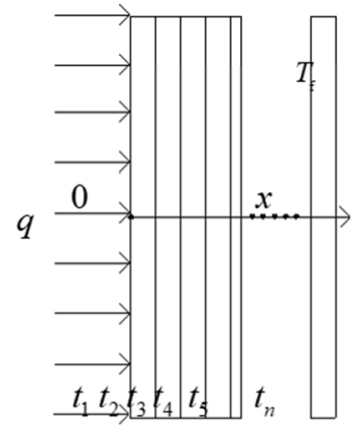

(a)unsteady heat conduction of N-layer wall

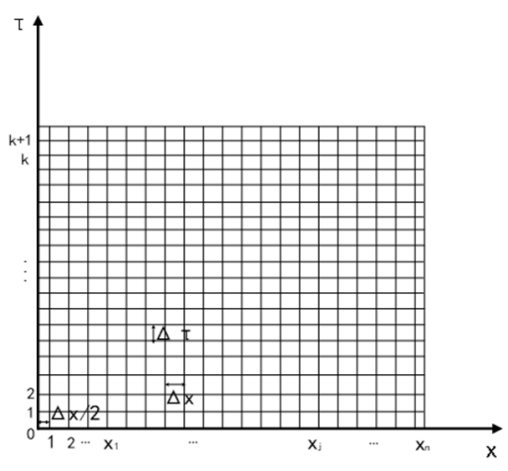

(b) unsteady heat conduction of finite difference method for mesh generation of Nlayer wall

Fig. 2. Finite difference method for assembled lightweight sandwich plates

The heat conduction differential equation of the plate can be expressed as

$$
\frac{\partial T_{\mathrm{j}}}{\partial \tau}=\alpha_{i} \frac{\partial^{2} T_{\mathrm{j}}}{\partial x^{2}}\left(x_{\mathrm{j}} \leq x \leq x_{\mathrm{j}+1}, \mathrm{j}=1,2 \cdots, n\right) .
$$

The Taylor series expansion is used to discretize the differential equations of the plate, which can be obtained by using the center difference method

$$
\frac{T_{\mathrm{j}, \mathrm{i}}^{\mathrm{k}+1}-T_{\mathrm{j}}^{\mathrm{k}+1}}{\Delta \tau}=\alpha_{j} \frac{T_{\mathrm{j}, \mathrm{i}+1}^{\mathrm{k}+1}-2 T_{\mathrm{j}, \mathrm{i}}^{\mathrm{k}+1}-T_{\mathrm{j}, \mathrm{i}-1}^{\mathrm{k}+1}}{(\Delta x)^{2}}\left(x_{\mathrm{j}} \leq x \leq x_{\mathrm{j}+1}, \mathrm{j}=1,2 \cdots, n\right) .
$$

The third boundary condition of the outer wall surface by solar radiation:

$$
q=-\left.\lambda_{1} \frac{\partial T_{1}}{\partial x}\right|_{x=0} .
$$

The second boundary condition of the outer wall surface and ambient temperature

$$
-\left.\lambda_{\mathrm{n}+1} \frac{\partial T_{\mathrm{n}+1}}{\partial x}\right|_{x=x_{\mathrm{n}+1}}=h\left(T_{\mathrm{n}+1}-T_{\mathrm{f}}\right) .
$$

Heat flow balance at the junction of different material in the $x_{\mathrm{j}}$ wall surface

$$
\lambda_{\mathrm{j}} \frac{\partial T_{\mathrm{j}}}{\partial x}=\lambda_{\mathrm{j}+1} \frac{\partial T_{\mathrm{j}+1}}{\partial x}\left(x=x_{\mathrm{j}}, \mathrm{j}=1,2 \cdots \cdot, n\right)
$$

Temperature balance at the junction of different material in the $x_{\mathrm{j}}$ wall surface

$$
T_{\mathrm{j}}=T_{\mathrm{j}+1}\left(x=x_{\mathrm{j}}, \mathrm{j}=1,2 \cdots \cdot, n\right) .
$$

The Taylor series expansion is used to discretize the boundary condition of the plate, internal boundary layer condition can be expressed as:

at point $x=x_{\mathrm{j}}$, it is assumed that we can use $i=x_{\mathrm{j}}+1$ by forward difference method to obtain the node

$$
\mathrm{T}_{\mathrm{j}, \mathrm{x}_{\mathrm{j}}}^{\mathrm{k}}=-\frac{\lambda_{\mathrm{j}+1}}{\lambda_{\mathrm{j}}} \mathrm{F}_{\mathrm{o}, \mathrm{j}} \mathrm{T}_{\mathrm{j}+1, \mathrm{x}_{\mathrm{j}}+1}^{\mathrm{k}+1}+\left(1+\mathrm{F}_{\mathrm{o}, \mathrm{j}}+\frac{\lambda_{\mathrm{j}}}{\lambda_{\mathrm{j}+1}} \mathrm{~F}_{\mathrm{o}, \mathrm{j}}\right) \mathrm{T}_{\mathrm{j}+1, \mathrm{x}_{\mathrm{j}}}^{\mathrm{k}+1}-\mathrm{F}_{\mathrm{o}, \mathrm{j}} \mathrm{T}_{\mathrm{j}, \mathrm{x}_{\mathrm{j}}-1}^{\mathrm{k}+1},(9)
$$

outer boundary layer condition can be expressed as: according to the unit balanced method, the quantity of heat through the object is equal to the increase of the internal energy in unit time, discretization of the second boundary condition can be expressed as

$$
\lambda_{\mathrm{n}} \frac{\mathrm{T}_{\mathrm{n}, \mathrm{x}_{\mathrm{n}}-1}^{\mathrm{k}+1}-\mathrm{T}_{\mathrm{n}, \mathrm{x}_{\mathrm{n}}}^{\mathrm{k}+1}}{\Delta \mathrm{x}}+\mathrm{h}\left(\mathrm{T}_{\mathrm{f}}-\mathrm{T}_{\mathrm{n}, \mathrm{x}_{\mathrm{n}}}^{\mathrm{k}+1}\right)=\rho_{\mathrm{n}} \mathrm{c}_{\mathrm{n}} \frac{\Delta \mathrm{x}}{2} \frac{\mathrm{T}_{\mathrm{n}, \mathrm{x}_{\mathrm{n}}}^{\mathrm{k}+1}-\mathrm{T}_{\mathrm{n}, \mathrm{x}_{\mathrm{n}}}^{\mathrm{k}}}{\Delta \tau}
$$

discretization of the third boundary condition for constant heat flux q can be expressed as

$$
\lambda_{1} \frac{T_{1,1}^{\mathrm{k}+1}-T_{1,0}^{\mathrm{k}+1}}{\Delta x}+q=\rho_{1} c_{1} \frac{\Delta x}{2} \frac{T_{1,0}^{\mathrm{k}+1}-T_{1,0}^{\mathrm{k}}}{\Delta \tau} .
$$

According to the finite difference scheme of the N-layer 
wall, the finite difference method is used to solve the numerical solution of the unsteady heat conduction of the N-layer wall by Matlab software. Firstly, we input the external boundary conditions of the environment and the basic information of each layer plate. Secondly, we conducted mesh generation along the time axis and thickness direction. Finally, we establish matrix equation to obtain the result by using the chasing method. Then select the output time, the response of temperature of each layer plate, and entering the drawing module to select the appropriate drawing mode for drawing. The flow chart is shown in Fig. 3.

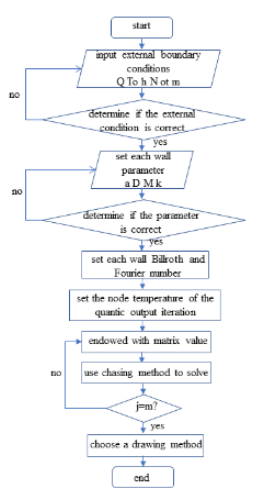

Fig. 3. Flow chart of one-dimensional unsteady heat conductivity coding

\section{The response of the defective surface temperature difference in different situations}

Taking the defect of $5 \mathrm{~mm}$ thickness as an example, polyurethane foam is used as the defective material to simulate the internal empty drum, and the thermophysical parameters of the material are shown in Table 1. As shown in Fig. 4, the feasibility study of the defect thickness, defect position, external heating strength, external heating time, and defects under defects was carried out by using the pre-programming Matlab program.

Table 1. Thermal and physical parameters of sandwich board

\begin{tabular}{ccccc}
\hline Physical parameters & $\lambda\left(\mathrm{w} / \mathrm{m} \cdot{ }^{\circ} \mathrm{C}\right)$ & $\mathrm{c}(\mathrm{kJ} / \mathrm{kg} \cdot \mathrm{K})$ & $\rho\left(\mathrm{kg} \cdot \mathrm{m}^{3}\right)$ & $\alpha\left(\mathrm{m}^{2} \cdot \mathrm{s}\right) \times 10^{7}$ \\
\hline Concrete slab & 1.54 & 0.84 & 2400 & 7.6 \\
Polyurethane foam & 0.033 & 1.38 & 30 & 8.0 \\
Rock wool & 0.038 & 0.75 & 140 & 3.6 \\
\hline
\end{tabular}

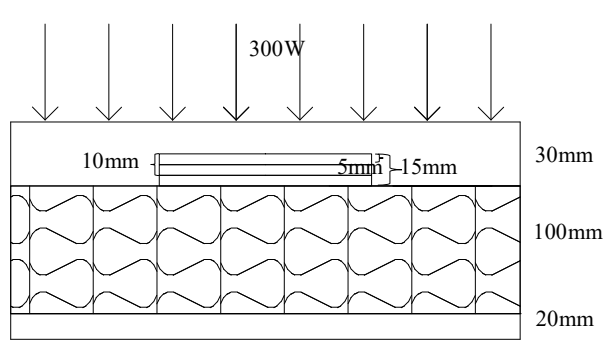

(a)different thickness

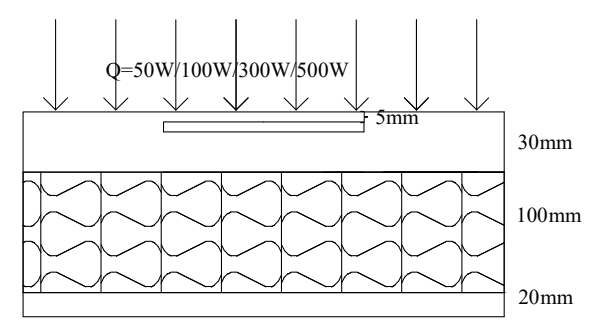

(c)different thermal current intensity and heating time

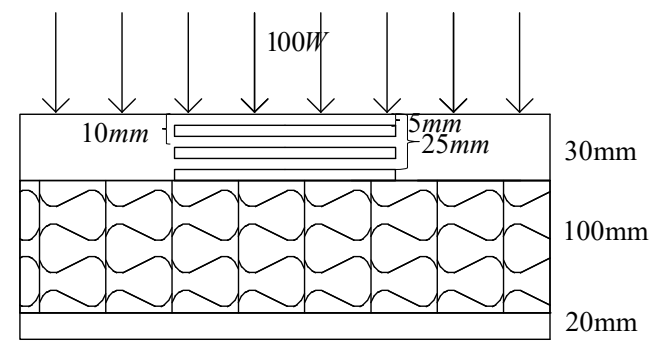

(b)different position

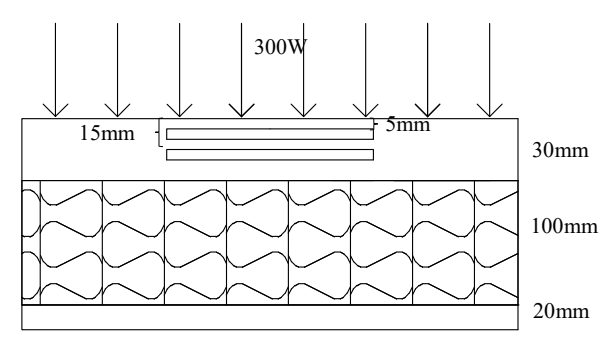

(d)defects under defects

Fig. 4. Different kinds of defects 
From Fig. 5(a), the surface temperature of different thickness defects and the surface temperature of the flawless defect, the thickness increased, hence, the surface temperature was higher under the constant heat flow with the same heating time. The $2^{\circ} \mathrm{C}$ temperature difference could be generated by heating at $300 \mathrm{~W}$ for 15 minutes. But from Fig. 5(b), in the first 20 minutes of heating, thickness made little impact on the temperature difference of defects. With the heating time increased, the surface temperature difference of defects in the same position with different thickness was more and more obvious. From Fig. 5(c), the surface temperature of different defect position and the surface temperature of flawless defect, the depth of defect position increased, hence, the surface temperature was lower under the constant heat flow with the same heating time. The $2^{\circ} \mathrm{C}$ surface temperature difference could be generated by heating at $100 \mathrm{~W}$ for 10 minutes in $5 \mathrm{~mm}$ thickness defect. However, the $2^{\circ} \mathrm{C}$ surface temperature difference could be generated by heating at $100 \mathrm{~W}$ for 40 minutes in $15 \mathrm{~mm}$ thickness defect. Less than $1{ }^{\circ} \mathrm{C}$ surface temperature difference could be generated by heating at $100 \mathrm{~W}$ for 60 minutes in $25 \mathrm{~mm}$ thickness defect which is the juncture of the concrete slab and rock wool. From Fig. 5(d), the heating time increased, hence, the growth rate of the surface temperature

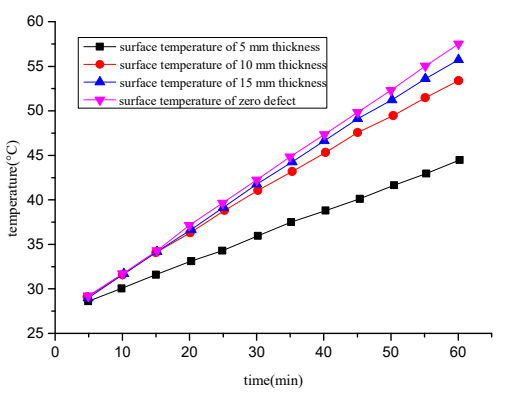

(a) the surface temperature of different thickness

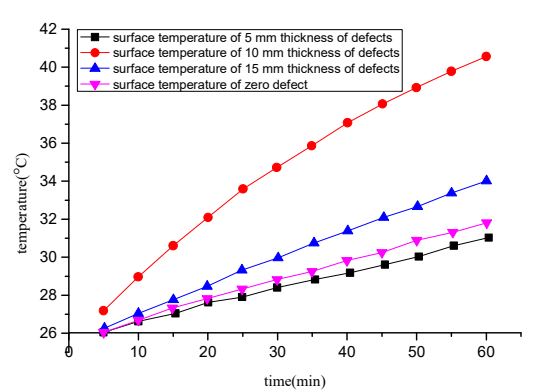

(c) the surface temperature of different position difference of defects was lower. From Fig. 6(e), the heating intensity increased, hence, the surface temperature difference of defects was greater under the same position with the same time. From Fig. 5(f), the heating time increased, hence, the growth of the surface temperature difference of defects was slower. The $2^{\circ} \mathrm{C}$ surface temperature difference could be generated by heating at $50 \mathrm{~W}$ for 20 minutes in $5 \mathrm{~mm}$ thickness defect. The $2^{\circ} \mathrm{C}$ surface temperature difference could be generated by heating at $100 \mathrm{~W}$ for 10 minutes in $5 \mathrm{~mm}$ thickness defect. The $2{ }^{\circ} \mathrm{C}$ surface temperature difference could be generated by heating at $300 \mathrm{~W}$ and $500 \mathrm{~W}$ for less than 5 minutes in $5 \mathrm{~mm}$ thickness defect. In actual construction sites, the suitable heating intensity could make great impact on reducing the heating time to save the time of detection. As shown in Fig. 5(g)-5(h), Comparative analysis was performed from one plate defect in $5 \mathrm{~mm}$ thickness and the other plate defect was at $10 \mathrm{~mm}$ lower distance of it, and under the condition of the same heat flow, the surface temperature of defects under defects was almost coincident with flawless defect curve in the early stage. With the increase of the heating time, the growth ratio of surface temperature difference of flawless defect surface is faster than the growth ratio of surface temperature difference of defects under defects.

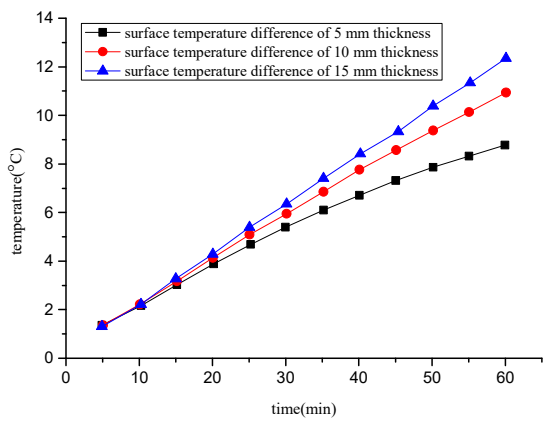

(b) the surface temperature difference of different thickness

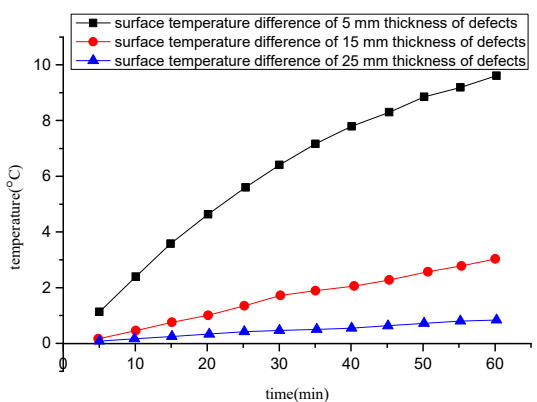

(d) the surface temperature difference of different position 


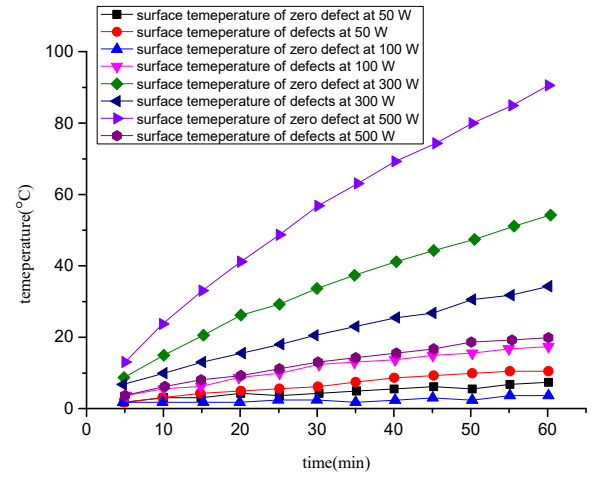

(e) the surface temperature of different heat flow

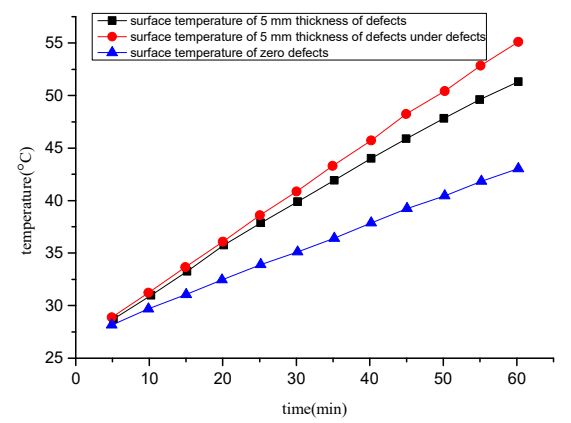

(g) the surface temperature of defects under defects

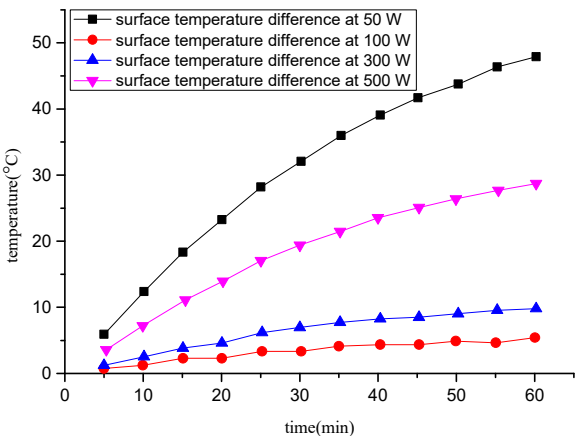

(f) the surface temperature difference of different heat flow

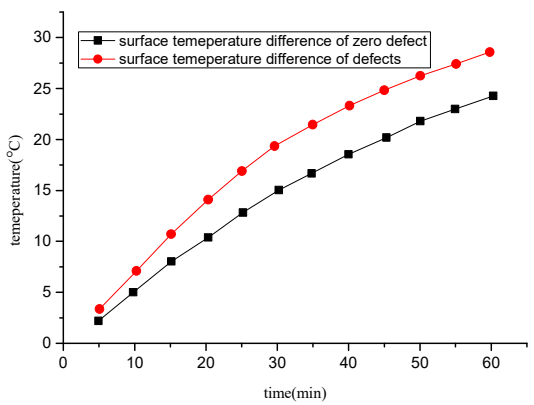

(h) the surface temperature difference of defects under defects

Fig. 5. Numerical results of defects under different types

\section{The verification on the feasibility of infrared thermal imaging}

Figure 6 shows the defects of $5 \mathrm{~mm}$ thickness in the position of $5 \mathrm{~mm}, 10 \mathrm{~mm}, 15 \mathrm{~mm}$ and $20 \mathrm{~mm}$ respectively. From the programming data, the surface temperature of flawless defect $(0 \mathrm{~mm})$ was $43^{\circ} \mathrm{C}$, and the surface temperature of the $5 \mathrm{~mm}$ defect was $67^{\circ} \mathrm{C}$, and the surface temperature of the $10 \mathrm{~mm}$ defect was $54^{\circ} \mathrm{C}$, and the surface temperature of the $15 \mathrm{~mm}$ defect is $48^{\circ} \mathrm{C}$, and the surface temperature of the $20 \mathrm{~mm}$ defect is $43^{\circ} \mathrm{C}$. We found that the defect closer to the surface, the surface temperature difference was greater. However, if defects in rock wool (in the position of $20 \mathrm{~mm}$ ), the surface temperature difference is almost same as the flawless defect rock wool. It is impossible to detect the rock wool defects with temperature difference ${ }^{[6]}$. The position of $15 \mathrm{~mm}$ defect was located in the juncture of rock wool and concrete plate. It could be seen the surface temperature difference was $5^{\circ} \mathrm{C}$ at that time. The temperature measurement accuracy of the infrared thermography was about $\pm 2{ }^{\circ} \mathrm{C}$. It is feasible to detect the felting defects of the interface of the lightweight sandwich plate and the compactness of the plate by using infrared thermography detection ${ }^{[7]}$.

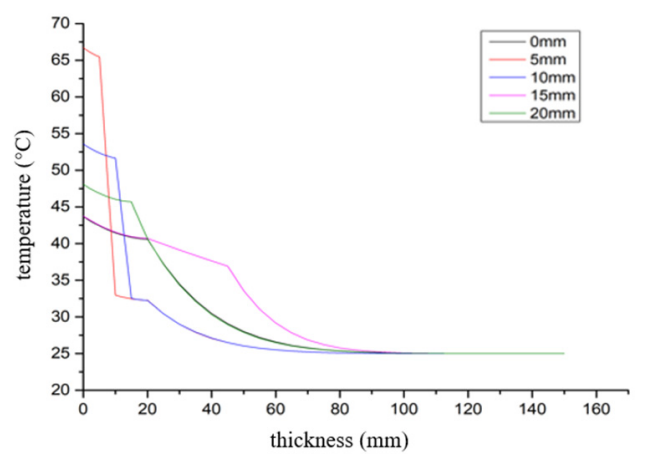

Fig. 6. Unsteady thermal conductivity temperature response of the sandwich plate at different defects

\section{Conclusion}

A finite difference method program for unsteady heat conduction is compiled with MATLAB software. Since the main defect of the analysis is plate defect, the defective prefabricated lightweight sandwich plate can be analyzed on the basis of approximately regarding the defect layer as a layer of defective plate. Numerical calculations are carried out mainly from the thickness of defects, the position of defects, external heat flow, heating time, and defects under defects. It is better to use infrared thermal 
imaging detection with more than $300 \mathrm{~W}$ heat source to save the time of detection based on the previous discussion about heating intensity and heating time. According to the surface temperature difference generated by the heating time, the position of defects is approximately determined. Due to the variation of surface temperature with heating time, the thickness of defects is approximately determined. Given that the growth ratio of surface temperature difference with time, the result of the numerical calculations of defects under defects shows that the defects are approximately determined. Above all, accurate detection can be achieved in infrared thermography detection under $2^{\circ} \mathrm{C}$ temperature difference situation. With the result of numerical calculations, it is feasible to use the infrared thermal imaging to detect the prefabricated lightweight plate. According to the processing technology of the prefabricated lightweight sandwich plate and the surface temperature difference, the position of the defect is approximately determined which provides a basis for future processing improvement.

\section{Acknowledgment}

This study was founded by the National Natural Science Foundation of China (No 51578181) , Natural
Science Foundation of Guangdong Province ( No 2016A030313665). The authors would like to extend their appreciation to Shenzhen Carbon Storage Cementbased Materials Engineering Laboratory

\section{References}

1. J. Hoła, K. Schabowicz, Arch. Civ. Mech. Eng. 10(3), 5-18, (2010)

2. S.M. Yang, W.Q. Tao, Heat transfer (Higher Education Press, Beijing, 1998)

3. D.R. Green, Appl. Optics. 7(9), 71-79, (1968)

4. H.H. Zhang, Sci. Technol. Inf. 15(3), 18-26, (2013)

5. J.T. Jiang, H.F. Xu, J.F. Wu, Nondestructive. Test. 37(10), 39-46, (2015)

6. H.R. Hua, J. Xiong, L.H. Yuan, J. NCHU (NAT. SCI). 2, 93-97, (2015)

7. C.Q. Wu, X.H. Hong, W.P. Wang, Q.G. Yuan, Y.J. Li, W. Zhang. High. Power. Laser. Part. Beam. 23(12), 3271-3274, (2011) 